\title{
Blow-up phenomena in a class of coupled reaction-diffusion system with nonlocal boundary conditions
}

\author{
Huimin Tian ${ }^{1}$, Lingling Zhang ${ }^{1}$, and Xin Wang ${ }^{1}$ \\ ${ }^{1}$ Taiyuan University of Technology
}

September 4, 2020

\begin{abstract}
The paper deals with blow-up phenomena for the following coupled reaction-diffusion system with nonlocal boundary conditions: $\backslash$ begin $\{$ equation* $\} \backslash$ left $\backslash\left\{\backslash\right.$ begin $\{$ aligned $\}$ \&u_t= $=$ nabla $\backslash$ cdot $\backslash$ big $(\backslash$ rho_1(u) $\backslash$ nabla u $\backslash$ big $)+a_{-} 1(\mathrm{x}) f_{-} 1(\mathrm{v}), \sim^{\sim}{ }_{\text {v_t }}=\backslash$ nabla $\backslash$ cdot $\backslash$ big $(\backslash$ rho_-

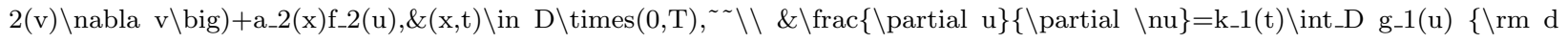
\} $\mathrm{x}, \sim \sim$ frac $\{\backslash$ partial v $\}\{\backslash$ partial $\backslash \mathrm{nu}\}=\mathrm{k} \_2(\mathrm{t}) \backslash$ int_D g_2(v) $\{\backslash \mathrm{rm} \mathrm{d}\} \mathrm{x}, \&(\mathrm{x}, \mathrm{t}) \backslash$ in $\backslash$ partial $\mathrm{D} \backslash \operatorname{times}(0, \mathrm{~T}), \backslash \backslash \& \mathrm{u}(\mathrm{x}, 0)=\mathrm{u} \_0(\mathrm{x}), \sim \sim \mathrm{v}(\mathrm{x}, 0)=\mathrm{v} \_-$

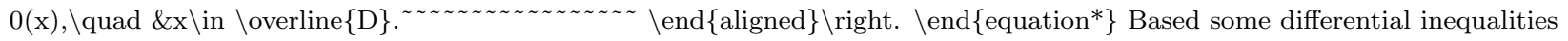
and Sobolev inequality, we establish conditions on the data to guarantee the occurrence of the blow-up. Moreover, when the blow-up occurs, explicit lower and upper bounds on blow-up time are obtained. At last, an example is presented to illustrate our main results.
\end{abstract}

\section{Hosted file}

\begin\{CJK\}\{UTF8\}\{gbsn\}. \end\{CJK\}\selectlanguage\{english\}pdf available at https://authorea. } com/users/356177/articles/479149-blow-up-phenomena-in-a-class-of-coupled-reactiondiffusion-system-with-nonlocal-boundary-conditions 